\title{
Resolution Uniformity and Sensitivity of the NIH ATLAS Small Animal PET Scanner: Comparison to Simulated LSO Scanners Without Depth-of-Interaction Capability
}

\author{
Jürgen Seidel, Member, IEEE, Juan José Vaquero, Senior Member, IEEE, and Michael V. Green
}

\begin{abstract}
Positron emission tomography (PET) scanners designed to image animals the size of rats and mice should possess simultaneously high and uniform spatial resolution and high sensitivity. ATLAS (Advanced Technology Laboratory Animal Scanner), a $6.0 \mathrm{~cm}$ diameter effective transverse field-of-view (FOV), $2 \mathrm{~cm}$ axial FOV ring-type research scanner seeks these goals by surrounding the animal with eighteen $15 \mathrm{~mm}$ deep, LGSO $(7 \mathrm{~mm}) / \mathrm{GSO}(8 \mathrm{~mm})$ phoswich detector modules.

A Monte Carlo simulation was used to compare the variation of resolution across the FOV and the absolute central point source sensitivity (ACS) of ATLAS to similar systems comprised only of LSO arrays of different depths with no depth-of-interaction (DOI) capability. For ATLAS radial spatial resolution deteriorated by $27 \%$ from the center to $3 \mathrm{~cm}$ off-axis. Scanners comprised of 15 mm deep, $10 \mathrm{~mm}$ deep and $7 \mathrm{~mm}$ deep LSO crystals deteriorated by $100 \%, 51 \%$, and $20 \%$, respectively, over the same distance. Simulated ACS (absorbed energies $\geq 250 \mathrm{keV}$ ) for ATLAS was $2.0 \%$ and for the $15 \mathrm{~mm}, 10 \mathrm{~mm}$ deep and $7 \mathrm{~mm}$ deep LSO scanners $2.4 \%, 1.5 \%$, and $0.9 \%$, respectively.

Radial resolution loss $3 \mathrm{~cm}$ off-axis and ACS measured for the actual ATLAS scanner were similar to the values obtained by simulation (27\% resolution loss, $1.8 \%$ ACS). The phoswich design thus achieves good resolution uniformity over a $6 \mathrm{~cm}$ FOV while preserving sensitivity compared to equivalent non-DOI LSO scanners with a range of crystal depths.
\end{abstract}

Index Terms-Depth-of-interaction, phoswich detectors, positron emission tomography (PET), small animal PET.

\section{INTRODUCTION}

A SMALL animal PET scanner should have high sensitivity and high and uniform spatial resolution across a field-of-view (FOV) larger than the largest object to be imaged. We have sought to meet these requirements by designing a small ring diameter, depth-of-interaction (DOI) PET scanner for imaging animals up to $6 \mathrm{~cm}$ in diameter. "ATLAS" (Advanced Technology Laboratory Animal Scanner) is based on "phoswich" detector modules constructed from two optically connected, but different, "fast" scintillator layers coupled to miniature position-sensitive photomultiplier tubes (PSPMT's). The scintillator-of-interaction is identified by "delayed charge

Manuscript received January 9, 2002; revised April 11, 2003.

J. Seidel and M. V. Green are with the National Institutes of Health, Bethesda, MD 20892 USA (e-mail: jurgen_seidel@nih.gov; mgreen@mail.cc.nih.gov).

J. J. Vaquero was with the National Institutes of Health, Bethesda, MD 20892 USA. He is now with the Hospital General Universitario Gregorio Marañón 28007 Madrid, Spain (e-mail: juanjo@mce.hggm.es).

Digital Object Identifier 10.1109/TNS.2003.817282 integration" (DCI), a method that effectively measures the decay time (LGSO: $40 \mathrm{~ns}$, GSO: $60 \mathrm{~ns}$ ) of the light pulse of each event [1]. With this technique it is possible to have a substantial total crystal depth $(15 \mathrm{~mm})$ while at the same time having apparently short crystals ( $7 \mathrm{~mm}$ LGSO and $8 \mathrm{~mm} \mathrm{GSO}$ ) that minimize the DOI effect at small ring diameters. The small ring diameter, in turn, increases sensitivity by increasing the system solid angle and reduces cost by reducing the number of detector modules needed to surround the animal.

While the ATLAS design exploits well-known principles for minimizing the DOI effect and increasing sensitivity, the degree to which this strategy achieves these goals compared to simpler schemes is also important. The construction of phoswich modules requires additional fabrication steps and necessitates other electronic and data processing changes that complicate the design of this machine. These complications could be avoided if a scanner without DOI capability and with only a single crystal layer could achieve the same performance. Accordingly, we utilized the Monte Carlo simulator created to study the characteristics of ATLAS to evaluate LSO scanners with identical ring diameters and axial FOV but having crystals of different depths and no DOI capability. We validated this simulation code by comparing the variation in radial spatial resolution and the absolute central point source sensitivity (ACS) determined for ATLAS by simulation with measured values obtained from the actual scanner.

\section{MATERIALS AND METHODS}

ATLAS (Fig. 1) consists of 18 LGSO/GSO (Hitachi) phoswich detector modules arranged around a ring $11.8 \mathrm{~cm}$ in diameter [2]. Each module is comprised of a $9 \times 9$ array of $2 \mathrm{~mm}$ square $\times 15 \mathrm{~mm}$ deep phoswich elements $(2.25 \mathrm{~mm}$ pitch), each of which is composed of a $7 \mathrm{~mm}$ long LGSO crystal optically glued end-on to an $8 \mathrm{~mm}$ long GSO crystal. The GSO end of the crystal bundle is optically glued to a miniature PSPMT (Hamamatsu R7600-C8).

ATLAS acquires coincidence events in 3-D (only) between a given detector modules and the seven opposite modules. The 18 detector modules are combined electrically in groups of three to form a total of six sectors. The signals originating from the ATLAS sectors are fed to a data acquisition system supplied by A \& D Precision Co. (Newton, MA). The system consists of six charge integrating ADC modules [3] with custom modifications 


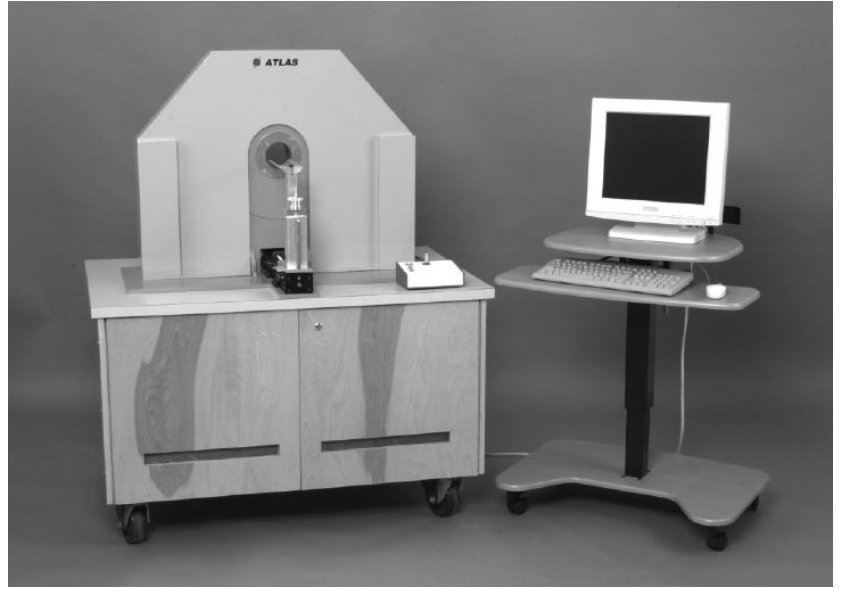

Fig. 1. The ATLAS small animal PET scanner.

to facilitate identification of scintillation decay times, one scaler module, a custom coincidence logic controller, and a high-speed PCI-bus interface card. The controller detects coincidences between sectors and initiates signal integration and ADC readout. Two alternating $128 \mathrm{kB}$ memory buffers on a PCI-bus card collect these data on a dual-processor PC operating under the Linux OS.

A Monte Carlo code was written to simulate the geometry and physical response of the ATLAS system to $511 \mathrm{keV}$ annihilation radiation. The simulation included modeling of the polygonal detector geometry as well as the effects of positron range (F-18), annihilation photon noncollinearity, and Compton scattering within scintillation crystals and between scintillator layers.

Linear attenuation coefficients for LSO and GSO were derived as a function of photon energy from atomic photon cross-section tables and the known atomic composition of the scintillators. Since the physical properties of LGSO differ only slightly from LSO, LGSO was treated like LSO in the simulations. The manufacturer specified the supplied LGSO as $\mathrm{Lu}_{1.8} \mathrm{Gd}_{0.2} \mathrm{SiO}_{5}$, i.e., only $10 \%$ of the $\mathrm{Lu}$ atoms (atomic number $\mathrm{Z}=71)$ are replaced by $\mathrm{Gd}$ atoms $(\mathrm{Z}=64)$. As a result, the effective atomic number for LGSO is essentially the same as LSO. Our measurements of physical density (7.23 $\mathrm{gm} / \mathrm{cc}$ ) and scintillation efficiency (not reported) of LGSO were also similar to values reported for LSO $(7.4 \mathrm{gm} / \mathrm{cc})$.

The $0.25 \mathrm{~mm}$ gaps between phoswich elements were not modeled. Instead, in order to simplify the simulation, an array was treated as a continuous block of $2.25 \mathrm{~mm}$ wide crystals each directly touching its neighbors. This strategy causes ACS to be overestimated and radial resolution to be slightly underestimated. Sensitivity values were corrected for this effect by reducing the simulated ACS values by the product of the geometric fill-factors of the real arrays. No corrections were applied to the radial resolution estimates since their primary purpose here was to allow comparison of systems rather than accurately determine their absolute spatial resolution.

The polygonal ATLAS detector geometry exhibits small gaps between detector modules. As a consequence, small gaps are present in the sinograms that are compensated for by using the constrained Fourier space method [4].
TABLE I

SIMULATION PARAMETERS

\begin{tabular}{cc}
\hline \multicolumn{2}{c}{ All Systems } \\
\hline Ring Geometry & Eighteen-sided polygon \\
Ring Diameter & $11.8 \mathrm{~cm}$ \\
Axial Field-of-View & $2.0 \mathrm{~cm}$ \\
Gantry Aperture Diameter & $8.0 \mathrm{~cm}$ \\
Effective Transverse Field-of-View & $6.0 \mathrm{~cm}$ \\
Number of Detector Modules & 18 \\
Crystal Array Dimensions per Modufe & $20 \mathrm{~mm} \times 20 \mathrm{~mm}$ \\
Array Size & $9 \times 9$ \\
Crystal Pitch & $2.25 \mathrm{~mm}$ \\
Crystal Cross-Section & $2 \mathrm{~mm} \times 2 \mathrm{~mm}$
\end{tabular}

ATLAS only (simulated and actual)

\begin{tabular}{|c|c|}
\hline Type of Detector Module & Dual scintillator phoswich \\
\hline Phoswich Type and Lengths & $\begin{array}{c}\operatorname{LGSO}(7 \mathrm{~mm}) / \mathrm{GSO}(8 \mathrm{~mm})=15 \\
\mathrm{~mm} \text { total }\end{array}$ \\
\hline Number of Crystals per Module & $9 \times 9 \times 2$ layers $=162$ \\
\hline Total Number of Crystals & 2916 \\
\hline Scintillator Light Decay Times & $\mathrm{LGSO}=40 \mathrm{~ns}, \mathrm{GSO}=60 \mathrm{~ns}$ \\
\hline Depth Determination Method & Delayed charge integration \\
\hline \multicolumn{2}{|c|}{ LSO Scanners (simulated only) } \\
\hline Type of Detector Module & Single scintillator - no DOI \\
\hline Number of Crystals per Module & $9 \times 9=81$ \\
\hline LSO Crystal Lengths & $7 \mathrm{~mm}, 10 \mathrm{~mm}, 15 \mathrm{~mm}$ \\
\hline Total Number of Crystals & 1458 (same for all) \\
\hline
\end{tabular}

Although ATLAS acquires data only in 3-D, for the purposes of this study only 2-D data (true slices and one ring difference cross-slices) were reconstructed into images using 2-D filtered backprojection and a ramp filter (Nyquist frequency cutoff). All data, real or simulated, were reconstructed into images using these same methods.

After modeling the ATLAS scanner, the simulation code was altered to model three LSO scanners that differed from the ATLAS design only in the depths of their scintillation crystals: $15 \mathrm{~mm}, 10 \mathrm{~mm}$ and $7 \mathrm{~mm}$ of LSO. The same point source data obtained for the real and simulated ATLAS scanners were then acquired for each of these simulated scanners. Note that in these LSO scanners, no DOI information is available. Parameters descriptive of the actual ATLAS scanner and important to the simulations are listed in Table I.

\section{A. Simulated Resolution Uniformity}

Coincidence data were acquired from a simulated point source of $511 \mathrm{keV}$ annihilation radiation moved in $1 \mathrm{~mm}$ increments from the geometric center of the system to 32 $\mathrm{mm}$ off the central axis. Events were considered valid if both absorbed photons deposited more than $250 \mathrm{keV}$ in their respective detectors. Coincidences occurring between LGSO-LGSO crystals, LGSO-GSO crystals and GSO-GSO crystals were binned into separate sinograms. 


\section{B. Measured Resolution Uniformity}

The variation in resolution with radius for events depositing more than $250 \mathrm{keV}$ in each detector was measured for the actual ATLAS scanner using a $0.5 \mathrm{~mm}$ diameter Na-22 point source (Isotope Products Laboratories, Burbank, CA) at the center of a $25 \mathrm{~mm}$ diameter solid plastic ball. The source was imaged every two millimeters out to $31.3 \mathrm{~mm}$ starting $1.3 \mathrm{~mm}$ from the aperture center. Images of the source at each position were reconstructed in the same manner as in the simulation.

Radial and tangential spatial resolutions were calculated from the apparent width (FWHM) of the point sources in all data sets as a function of radial position. The width of the pixels in the reconstructed images was $0.28 \mathrm{~mm}$ and thus was considered small enough to have a negligible effect on the point source FWHM. FWHM in each direction was determined by fitting a 2-D Gaussian to the image of each source within a $5.3 \mathrm{~mm} \times$ $5.3 \mathrm{~mm}$ region bounding each source.

\section{Simulated Absolute Central Point Source Sensitivity}

The fraction of annihilations yielding detected coincidence events was determined for a point source located at the geometric center of each simulated scanner for energy thresholds of 100 and $250 \mathrm{keV}$.

\section{Measured Central Point Source Sensitivity}

A 1.2 mm-diameter F-18 line source two centimeters long and surrounded by a $2 \mathrm{~mm}$ thick lucite annulus was placed colinearly with the central axis of the ATLAS scanner exactly spanning the axial FOV. Coincident events were acquired for $100-650 \mathrm{keV}$ and $250-650 \mathrm{keV}$ energy windows and the central point source sensitivity, ACS, calculated with the relation: ACS $=2 \times$ central line source sensitivity, where central line source sensitivity is the coincidence rate for the given energy condition divided by the positron annihilation rate in the $2 \mathrm{~cm}$ long line source.

\section{RESULTS}

Plots of radial resolution versus radius measured for the real and for the simulated ATLAS scanner are compared to similar curves obtained for the three simulated LSO scanners in Fig. 2. Absolute central point source sensitivity estimates are summarized in Table II.

Tangential resolution did not vary by more than $10 \%$ for any of the scanners over the central $6 \mathrm{~cm}$ FOV and these results are not shown. Forty-three percent of all coincidences were identified as LGSO-LGSO, $44 \%$ as LGSO-GSO, and $13 \%$ as GSO-GSO. These values were used as weighting factors to combine the three spatial resolution estimates obtained for the different coincidences into the single ATLAS radial resolution curve shown in Fig. 2.

\section{DISCUSSION}

The results portrayed in Fig. 2 and Table II suggest that ATLAS achieves simultaneously good sensitivity and resolution uniformity compared to equivalent single scintillator designs without DOI capability. While the $15 \mathrm{~mm}$ LSO scanner possesses higher sensitivity, and the $7 \mathrm{~mm}$ scanner slightly

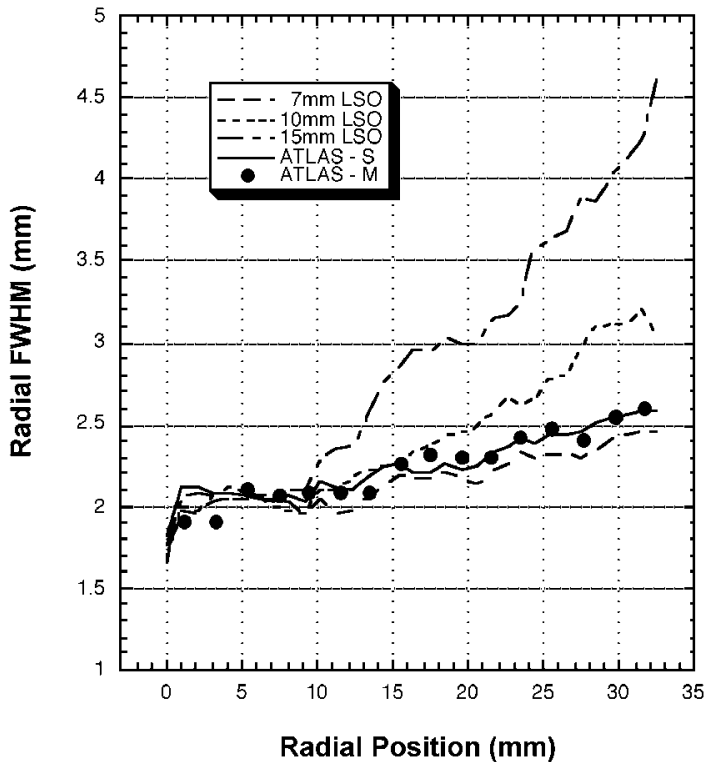

Fig. 2. Radial resolution versus radius for different scanners. ATLAS-S: simulated ATLAS results; ATLAS-M: measured ATLAS results.

TABLE II

Absolute Central Point Source Sensitivity

\begin{tabular}{|c|c|c|c|c|}
\hline $\begin{array}{c}\text { Threshold } \\
(\mathrm{keV})\end{array}$ & $\begin{array}{l}\mathrm{LSO} \\
7 \mathrm{~mm}\end{array}$ & $\begin{array}{c}\mathrm{LSO} \\
10 \mathrm{~mm}\end{array}$ & $\begin{array}{c}\text { LSO } \\
15 \mathrm{~mm}\end{array}$ & $\begin{array}{c}\text { ATLAS } 15 \mathrm{~mm} \\
\text { Phoswich }\end{array}$ \\
\hline & & & & $\mathrm{S}^{*} \quad \mathrm{M}^{*}$ \\
\hline 100 & $1.3 \%$ & $2.1 \%$ & $3.2 \%$ & $2.8 \% \quad 2.7 \%$ \\
\hline 250 & $0.9 \%$ & $1.5 \%$ & $2.4 \%$ & $2.0 \% \quad 1.8 \%$ \\
\hline
\end{tabular}

better resolution uniformity, neither of these devices matches ATLAS's sensitivity and resolution uniformity at the same time. The $10 \mathrm{~mm}$ LSO scanner, perhaps the best overall match to ATLAS, exhibits a $51 \%$ resolution variation $3 \mathrm{~cm}$ off-axis that is nearly a factor of two poorer than ATLAS (27\%) and is $25 \%$ less sensitive. MicroPET [5], an existing $17.2 \mathrm{~cm}$ diameter, $10 \mathrm{~mm}$ deep LSO scanner, exhibits the same spatial resolution at $3 \mathrm{~cm}$ as ATLAS, but with $0.6 \%$ sensitivity $(250-650 \mathrm{keV}$ energy window).

The simulated ATLAS radial resolution variation (curve $S$, Fig. 2) and ACS (Table II) are in close agreement with the measured resolution variation (M, Fig. 2) and ACS (Table II) of the actual scanner. These results illustrate the internal consistency of these data and support the validity of the simulation code.

Measured ACS for the full ATLAS ring (1.8\%) is slightly higher than our previous experimental estimate of ACS made on a partially completed ATLAS detector ring $(1.6 \%,[2])$. This difference can be attributed to the escape of positrons from the glass capillary tube $(0.2 \mathrm{~mm}$ wall thickness $)$ used in the previous study. Annihilation of these positrons takes place mostly outside the scanner's FOV and they remain undetected by the scanner whereas such events are usually included in the dose calibrator measurement. Our simulation shows that the additional $2 \mathrm{~mm}$ thick lucite annulus surrounding the line source leads to $11 \%$ 
more detectable annihilations, almost exactly the difference between the past [2] and present ACS measurements.

It is noteworthy that the present results are also in qualitative agreement with other experimental and simulation studies of DOI-capable scanners having similar geometry. MacDonald and Dahlbom [6] found experimentally that a two-layer phoswich improved radial resolution uniformity nearly as well as phoswich modules with a greater number of layers. Similarly, the simulation study by Rafecas et al. [7] showed that a two-layer phoswich could significantly reduce radial resolution degradation at small ring diameters while preserving sensitivity. Rafecas et al. also found the proportion of coincidence events occurring between the various phoswich layers to be nearly identical to those found in the present work when due account is given to the slight differences in crystal depth between studies, i.e., front-front coincidences $=40 \%$ [7] versus $43 \%$ (here), front-back coincidences $=46 \%$ [7] versus $44 \%$ (here) and back-back coincidences $=17 \%$ [7] versus $13 \%$ (here).

The absolute magnitude of spatial resolution shown in Fig. 2 and the apparent variation of resolution across the FOV depend, in part, on spatial sampling. The ATLAS phoswich design inherently increases by a factor of four the number of lines of response penetrating the imaging volume compared to geometrically similar, single crystal type, non-DOI scanners [5]. As a result, spatial sampling in both the transverse and axial directions is improved in ATLAS particularly for off-axis points. Spatial sampling in the central region of the transverse plane, however, remains relatively poor. To increase sampling in this region, the detector array in the actual ATLAS scanner can be mechanically "wobbled" (0.94 mm radius) at rates up to eight revolutions per second. Under such circumstances (which have yet to be fully evaluated) it is expected that resolution in the transverse plane in the central FOV can be improved by as much as $20 \%$. Other strategies to enhance spatial sampling (and spatial resolution) by more fully exploiting the unique geometry of the phoswich design are also under investigation.

It is also noteworthy that the delayed charge integration method can, in itself, improve spatial resolution by rejecting inaccurately positioned lines-of-response. Coincidences comprised of scatter events between the LGSO and GSO layers, for example, will contain a mixture of light decay times that differ from the exact decay times of LGSO and GSO. The endpoints of the lines-of-response associated with such events cannot be unambiguously associated with a crystal in either layer and thus may be incorrectly positioned. The DCI method can reject a large fraction of these events and thereby improve resolution. Similarly, careful identification of spatial regions-of-interest around each crystal center [8] can help eliminate intra-crystal scatter events. Additional studies are required to fully characterize these accuracy-enhancing rejection methods since these methods also reduce sensitivity.

Finally, coincidence data acquired by ATLAS will be reconstructed routinely using 3-D iterative, resolution recovery recon- struction algorithms [9] rather than 2-D or 3-D FBP. With such methods, it should be possible to capitalize on ATLAS's sensitivity and improve absolute spatial resolution substantially beyond that shown in Fig. 2.

\section{CONCLuSion}

The present study supports the view that the ATLAS design achieves simultaneously good resolution, resolution uniformity and sensitivity over a $6 \mathrm{~cm}$ FOV compared to non-DOI LSO scanners of similar aperture and axial FOV.

\section{ACKNOWLEDGMENT}

The authors would like to thank J. Sullivan, P. Fitze, J. Powell, F. Sharpnack II, and C. Toms, of the Mechanical Instrument Design and Fabrication Section, and B. Chidakel, Computer and Electronics Section, Office of Research Services, NIH, for their help with the ATLAS project. The authors would also like to thank R. Ford, Division of Engineering Services, NIH, and F. J. Barbosa and W. Gunning, Thomas Jefferson National Accelerator Facility. In addition, they gratefully acknowledge the assistance of V. Zavarzin, A \& D Precision Co., for customizing the data acquisition system to our specifications and G. Muehllehner for drawing their attention to the effect of positron escape on the measurement of ACS.

\section{REFERENCES}

[1] J. Seidel, J. J. Vaquero, S. Siegel, W. R. Gandler, and M. V. Green, "Depth identification accuracy of a three-layer phoswich PET detector module," IEEE Trans. Nucl. Sci., vol. 46, pp. 485-490, June 1999.

[2] J. Seidel, J. J. Vaquero, I. J. Lee, and M. V. Green, "Experimental estimates of the absolute sensitivity of a small animal PET scanner with depth-of-interaction capability," in Proc. Conf. Rec. 2000 IEEE Medical Imaging Conf., Lyon, France, paper M21-57.

[3] V. G. Zavarzin and W. A. Earle, "A 500k event/s 12-bit ADC system with high-speed buffered PCI interface," IEEE Trans. Nucl. Sci., vol. 4, pp. 414-416, June 1999.

[4] J. S. Karp, G. Muehllehner, D. A. Mankoff, C. E. Ordonez, J. M. Ollinger, M. E. Daube-Witherspoon, A. T. Haigh, and D. J. Beerbohm, "Continuous-slice PENN-PET: A positron tomograph with volume imaging capability," J. Nucl. Med., vol. 31, no. 5, pp. 617-627, 1990.

[5] A. F. Chatziioannou, S. R. Cherry, Y. Shao, R. W. Silverman, K. Meadors, T. H. Farquhar, M. Pedarsani, and M. E. Phelps, "Performance evaluation of MicroPET: A high-resolution Lutetium Oxyorthosilicate PET scanner for animal imaging," J. Nucl. Med., vol. 40, no. 7, pp. 1164-1175, 1999.

[6] L. R. MacDonald and M. Dahlbom, "Parallax correction in PET using depth of interaction information," IEEE Trans. Nucl. Sci., vol. 45, pp. 2232-2237, Aug. 1998.

[7] M. Rafecas, G. Böning, B. J. Pichler, E. Lorenz, M. Schwaiger, and S. I. Ziegler, "A Monte Carlo study of high resolution PET with granulated dual layer detectors," IEEE Trans. Nucl. Sci., vol. 48, pp. 1490-1495, Aug. 2001

[8] J. J. Vaquero, J. Seidel, S. Siegel, W. R. Gandler, and M. V. Green, "Performance characteristics of a compact position-sensitive LSO detector module," IEEE Trans. Med. Imaging, vol. 17, pp. 967-978, Dec. 1998.

[9] C. A. Johnson, J. Seidel, R. E. Carson, W. R. Gandler, A. Sofer, M. V. Green, and M. E. Daube-Witherspoon, "Evaluation of 3-D reconstruction algorithms for a small animal PET camera," IEEE Trans. Nucl. Sci., vol. 44, pp. 1303-1308, June 1997. 